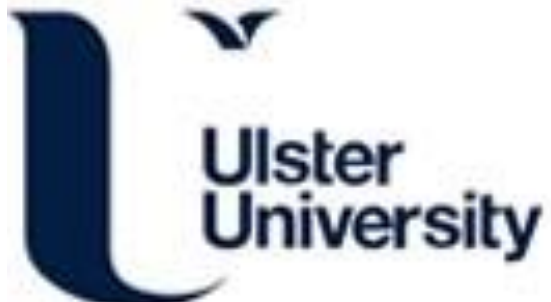

\section{mk-sense: An Extensible Platform to Conduct Multi-institutional Mobile Sensing Campaigns}

\begin{abstract}
Hernandez-Cruz, N., Arnrich, B., Favela, J., Gökhan, R., Ersoy, C., Demiray, B., \& Fontecha, J. (2016). m sense: An Extensible Platform to Conduct Multi-institutional Mobile Sensing Campaigns. Lecture Notes in Computer Science (including subseries Lecture Notes in Artificial Intelligence and Lecture Notes in Bioinformatics), 10069, 207-216. https://doi.org/10.1007/978-3-319-48746-5_21
\end{abstract}

Link to publication record in Ulster University Research Portal

\section{Published in:}

Lecture Notes in Computer Science (including subseries Lecture Notes in Artificial Intelligence and Lecture Notes in Bioinformatics)

\section{Publication Status:}

Published (in print/issue): 02/11/2016

DOI:

10.1007/978-3-319-48746-5_21

\section{Document Version}

Author Accepted version

\section{General rights}

Copyright for the publications made accessible via Ulster University's Research Portal is retained by the author(s) and / or other copyright owners and it is a condition of accessing these publications that users recognise and abide by the legal requirements associated with these rights.

\section{Take down policy}

The Research Portal is Ulster University's institutional repository that provides access to Ulster's research outputs. Every effort has been made to ensure that content in the Research Portal does not infringe any person's rights, or applicable UK laws. If you discover content in the Research Portal that you believe breaches copyright or violates any law, please contact pure-support@ulster.ac.uk. 


\title{
$\mathbf{m}^{\mathrm{k}}$-Sense: An Extensible Platform to Conduct Multi-institutional Mobile Sensing Campaigns
}

\author{
Netzahualcóyotl Hernández ${ }^{1(\varpi)}$, Bert Arnrich ${ }^{2}$, Jesús Favela ${ }^{1}$, Remzi Gökhan $^{2}$, \\ Cem Ersoy $^{2}$, Burcu Demiray ${ }^{3}$, and Jesús Fontecha ${ }^{4}$ \\ ${ }^{1}$ Computer Science Department, CICESE Research Center, Ensenada, Mexico \\ \{netzahdzc, favela\} @cicese.mx \\ ${ }^{2}$ Computer Engineering Department, Boğaziçi University, Istanbul, Turkey \\ \{bert.arnrich, gokhan.yavuz, ersoy\}@boun. edu.tr \\ ${ }^{3}$ Psychology Department, University of Zürich, Zurich, Switzerland \\ b.demiray@psychologie.uzh.ch \\ ${ }^{4}$ Computer Science Department, University of Castilla-La Mancha, Ciudad Real, Spain \\ jesus.fontecha@uclm.es
}

\begin{abstract}
Mobile sensing has become a growing area of research in pervasive healthcare. In this paper we present $\mathrm{m}^{\mathrm{k}}$-sense, an open framework for mobile sensing on smartphones. $\mathrm{m}^{\mathrm{k}}$-sense is an initiative to reduce the efforts of researchers involved in multi-institutional sensing campaign. It is designed to facilitate the collaboration of researchers that run simultaneous data collection efforts in different locations. We illustrate the use of $\mathrm{m}^{\mathrm{k}}$-sense with two cross-cultural studies conducted in four different countries (Turkey, Mexico, Switzerland, and Spain) with a total of 77 participants. In this paper, we describe the challenges and experience of conducting research in the wild by using $\mathrm{m}^{\mathrm{k}}$-sense as sensing platform. Finally, we present how the conducted studies influenced the design decisions of $\mathrm{m}^{\mathrm{k}}$-sense, including features, and tools to monitor data gathering in real-time.
\end{abstract}

Keywords: Mobile devices · Smartphones · Sensing campaign · Data management application

\section{Introduction}

Mobile phones are becoming pervasive worldwide. It has been estimated that there is an average of almost 2 mobile devices per user. Mobile phones have become the fastestselling gadgets, outselling computers four to one [1]. Moreover, Google announced it had hit over 1.4 billion monthly active users on the Android platform [2], with a tendency to further increase.

The proliferation of smartphones enables the gathering of user information from devices that are geographically scattered. This phenomenon gives researchers the opportunity to expand their understanding in many fields. For instance, Silva, T.H. et al., explored the potential of participatory sensing derived from location sharing systems (e.g., Foursquare) to understand human dynamics of cities [3]. Eagle, N. et al., used smartphones' Bluetooth as a proximity sensor, frequency of applications, and call 
records to recognize social patterns in the daily-life user activity, to infer relationships, and to identify socially significant locations [4].

Extracting collective information holds the potential to help us understand the dynamics of society, as well as to predict collective phenomena such the spread of an infectious disease. Examples are the observation of the spatiotemporal movements of millions of people during disease outbreaks [5], and the rapid detection of an unusual respiratory illness in a remote village [6].

There already exist sensing platforms to support data collection using smartphones. For instance, PRISM (Platform for Remote Sensing using Smartphones) allows to personalize a sensing application by its flexible use of pre-defined modules [7]; PHONELAB provides a manageable interface to initiate a sensing campaign with no coding involved [8]; MyExperience combines sensor and questionnaire collection of data among other functions [9]; FUNF consists of an open source framework to collect sensor data remotely and it provides services to define technical configuration at low level [10]. However, these studies have already tackled important aspects on a sensing campaign (i.e., scalability, no technical knowledge required, and variety on features such as sensor collection and surveys), few efforts have focused on providing a monitor service to supervise completeness of data along sensing campaigns, which is important in terms of quality and quantity of samples being collected.

\section{The Concept of $\mathbf{m}^{\mathrm{k}}$-Sense as a Platform}

$\mathrm{m}^{\mathrm{k}}$-sense is an initiative to reduce the efforts of researchers involved in multi-institutional sensing campaign. As a general view, $\mathrm{m}^{\mathrm{k}}$-sense implements a client-side application able to setup automatically based on server-side requests. It allows researchers to conduct simultaneous campaigns with minimal configuration effort. Thus, researchers could focus on their studies and gather data instead of investing resources in developing specific software.

In this paper, we describe $\mathrm{m}^{\mathrm{k}}$-sense, an extension of the open source framework Funf to collect data through mobile devices, and to manage the collaboration of multiple researchers involved in studies with the same approach. We present overviews of two studies conducted in naturalistic conditions in which we used our earliest version of the $\mathrm{m}^{\mathrm{k}}$-sense. We elaborate on how previous experiences influence the design of our final version of $\mathrm{m}^{\mathrm{k}}$-sense. We conclude with lessons learned, challenges, experience, and future work.

\section{$3 \mathbf{m}^{\mathrm{k}}$-Sense}

\subsection{Principles}

$\mathrm{m}^{\mathrm{k}}$-sense was designed based on the following principles:

- Approach centered. It is aimed at allowing researchers to focus on the analysis of the data rather than investing effort and resources on developing the sensing system. Thus, operating $\mathrm{m}^{\mathrm{k}}$-sense requires minimal technical knowledge. 
- Modular study-packages. It is based on the concept of packing, allowing researchers to create several studies simultaneously. Each package is treated independently, but managed with the same Graphical User Interface (GUI) environment.

- Creation of mobile phone campaign. Due to the purpose of the platform, after installing $\mathrm{m}^{\mathrm{k}}$-sense on a smartphone, participants are allowed to register themselves to any study-package available, as well as receive updates automatically, remotely and effortlessly.

\subsection{Architecture}

$\mathrm{m}^{\mathrm{k}}$-sense consists of a three-layer client-server architecture. The data-layer stores information about user devices, survey questions and responses, and sensor data in a relational database. The presentation-layer consists of a Graphical User Interface (GUI) that enables required services to create study-packages and to monitor data completeness. These two layers are executed in the server. Finally, the business-layer manages surveys and sensors, and temporarily keeps data in the client (i.e., smartphone) before it can be sent to the server.

As shown in Fig. 1, once the sensing application is installed on the smartphone, the user receives a sequence of surveys per day set up at random times (Fig. 1-a), while the smartphone constantly collects data through the sensors using Funf (Fig. 1-b). Both sensor and survey data are temporarily stored on the smartphone, waiting to be sent opportunistically by a wireless connection to the server. Batches of data are sent periodically to the server-side (Fig. 1-c). The server collects data and stores them in databases in a way that allows researchers to have an overview about the current study by providing a view of data completeness in real time (Fig. 1-d).
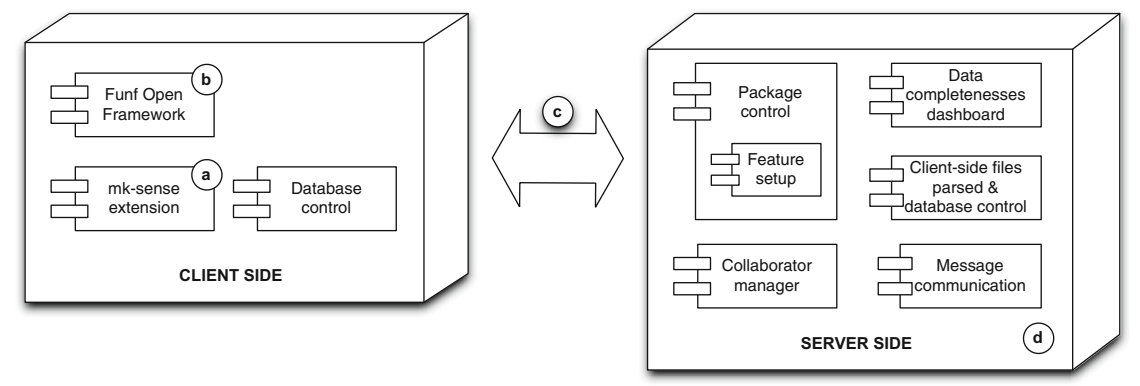

Fig. 1. $\mathrm{m}^{\mathrm{k}}$-sense data collection architecture.

Client. The client consists of an Android-based extensible framework designed to collect smartphone probes. It flexibility allows devices to be configured via server synchronization of all supported sensors from 3 different categories: (1) Sensor: accelerometer, Bluetooth devices, proximity and light sensor, gyroscope, WiFi scan, location, (2) Mobile phone: OS information, browser search history, cell towers, contact list, hardware information, on/off screen-event, phone-call logs, applications used, battery 
information, SMS history, running applications, and (3) Voluntary input: survey answers as self-report information, audio recording, and photo images.

Once $\mathrm{m}^{\mathrm{k}}$-sense is installed on the smartphone, data probes are collected in the background. Data is temporarily stored on the device as SQLite ${ }^{1}$ files, waiting to be sent periodically to the server; opportunistically and unobtrusively by $\mathrm{WiFi}$ connections.

Server. The server consists of a web system developed using the Laravel $^{2}$ framework and MVC (Model View Controller) architecture. The server constantly listens to requests from the smartphones to store data remotely. When data are successfully sent to the server, these files are deleted from the smartphone in order to release memory space on the mobile device. Meanwhile, the data received on the server are parsed and stored into a MySQL ${ }^{3}$ database. This is done by two different processes, so data are available to be monitored in real-time once received.

\subsection{Additional Features}

$\mathrm{m}^{\mathrm{k}}$-sense provides four additional features that distinguishes it from most sensing platforms: (1) audio recording is privacy-preserving, (2) photo collection, (3) questionnaires as surveys and its triggering function, and (4) dashboard.

- Audio recording. Audio data are a rich source of information, which allows, for example, to detect whether someone is having a conversation. On the other hand, most people do not accept audio recordings due to privacy reasons. In order to overcome this tradeoff, a privacy preserving way of audio recording was implemented. For each audio recording, the user can decide whether the recording is uploaded for further processing or deleted.

- Photo collection. Photographs are another rich source of information that allows a better understanding of the participant's context through a graphic representation.

- Surveys. $\mathrm{m}^{\mathrm{k}}$-sense supports two survey mechanisms: (1) experience sampling for gathering responses at random time intervals during a day, and (2) daily reconstruction survey for collecting responses at the end of the day after the last survey from the first category. Both types of surveys can consist of a mixture of input types: check boxes, sliders, free text entry and audio message.

- Dashboard. It is implemented in order to track the smartphones participating in a specific campaign. Sensor data are monitored through different visualization mechanisms, for instance to supervise accelerometer functionality and location data, as shown in Fig. 2.

To recognize data collection problems in a timely manner, a data completeness visualization is available. For instance, Fig. 3 shows accelerometer data collected for 9 days, segmented by hours; where the brightest intensity represents less data and the darkest intensity represents the highest amount of data collected in a period of time. It

\footnotetext{
${ }^{1}$ https://www.sqlite.org/.

${ }^{2}$ https://laravel.com/.

${ }^{3}$ https://www.mysql.com/.
} 


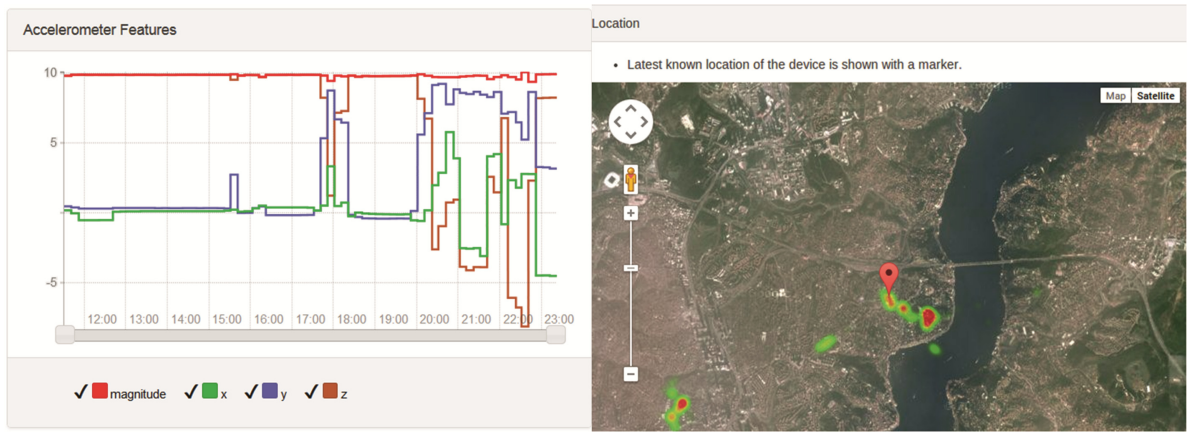

Fig. 2. Accelerometer (left) and location (right) visualizations available through dashboard.

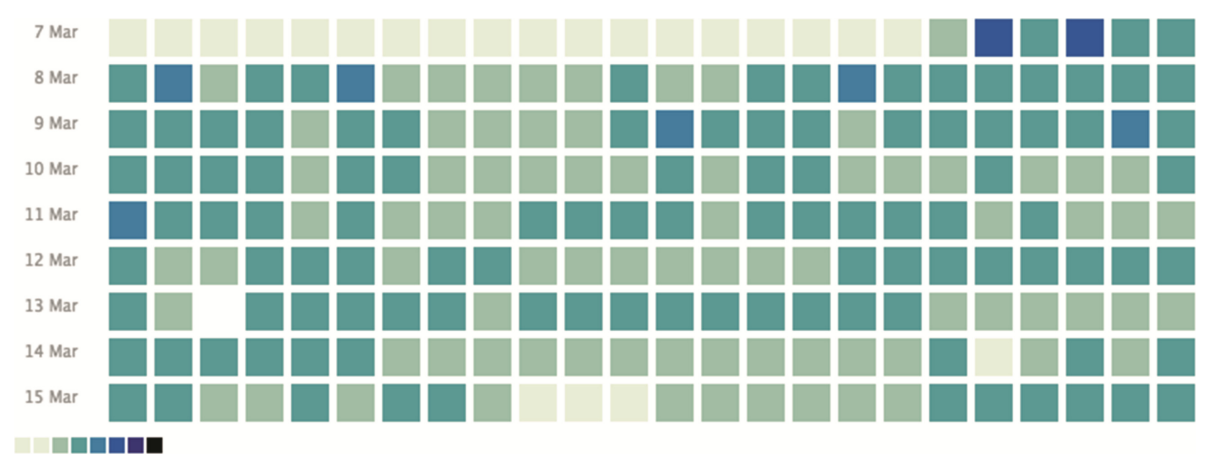

Fig. 3. Data completeness visualization of accelerometer data. Each line corresponds to one day and each square corresponds to one hour. Color-coding indicates the amount of data collected: bright color indicates no/less data; dark color stands for high amount of data. (Color figure online)

can be observed that data collection started in the evening of March $7^{\text {th }}$. In the afternoon of March $15^{\text {th }}$, no location data was collected for $3 \mathrm{~h}$. Afterwards, the data connection problem was solved and data collection resumed. Similarly, on March $13^{\text {rd }}$ and $14^{\text {th }}$ data was missed for one hour each day.

If the researcher detects a problem with data collection during the sensing campaign, he or she can inform the participant by sending a message. Messages are sent as notifications directly to the participants' smartphone. Alternatively, the system can be configured to send these messages automatically once a condition is detected.

\subsection{Study-Package Structure}

A study package follows the idea consists of the idea of enclosing a resource in a single virtual location to keep information well organized and easy to handle when performing any maintenance operation to respective data.

A package can be created by defining a list of sensors to be enabled in the study, a set of rules to define duty cycles, and triggering conditions. Study packages are automatically updated in every smartphone that has previously installed $\mathrm{m}^{\mathrm{k}}$-sense, reducing 
the time and effort to distribute any study campaigns to the participants. Thus, participants have the option to subscribe or unsubscribe from a set of available campaigns.

\section{Conducting Multi-site Sensing Campaigns}

As a proof of concept of the $\mathrm{m}^{\mathrm{k}}$-sense framework, we present multi-institutional sensing campaigns: (1) Thought and Life Logging (Tholilo) [11], and (2) a 21-day sensing campaign on walkability. Both projects were deployed in several sites using the betaversion of the $\mathrm{m}^{\mathrm{k}}$-sense platform.

\subsection{Thought and Life Logging (Tholilo)}

Tholilo is a collaborative research study that involves computer engineers and psychologists. The aim is to better interpret how daily life environments influence our mood and temporal thinking. Data were collected in two different locations: Turkey and Switzerland. Table 1 shows some basic characteristics of both samples. In order to keep the battery consumption low, sensor data were not collected continuously, but in periodic time intervals. The following sensor configuration was employed:

- Bluetooth (scans for devices every $5 \mathrm{~min}$ ),

- GPS (every 30 min),

- Accelerometer (sample with a frequency according to user's device configuration on intervals of $10 \mathrm{~s}$ every $5 \mathrm{~min}$ ),

- Running apps,

- Screen on/off states.

Table 1. Sample characteristics.

\begin{tabular}{l|l|l}
\hline & Group A & Group B \\
\hline No. of participants & 6 & 6 \\
\hline City, Country & Zürich, Switzerland & Istanbul, Turkey \\
\hline Size of the city & Medium $(396 \mathrm{k})$ & Large $(14 \mathrm{M})$ \\
\hline Density of population & $4.5 \mathrm{k} / \mathrm{km}^{2}$ & $2.6 \mathrm{k} / \mathrm{km}^{2}$ \\
\hline Gender & $(1 \mathrm{male} ; 5$ female $)$ & $(1 \mathrm{male} ; 5$ female $)$ \\
\hline Average age (S.D) & $33.16(14.40)$ & $23.33(1.5)$ \\
\hline
\end{tabular}

Each participant was asked to complete a survey 7 times a day at random time points. Right before each notification, a one-minute audio sample was recorded in the privacypreserving format explained earlier. Surveys focused on collecting data on momentary feelings and thoughts based on an already standardized protocol. Data were collected during a 10-day period.

The users carried their smartphones as usual, no special instructions were given to operate the system. We conducted the study with two different groups of participants. A total of 12 volunteers participated. The inclusion criteria were that the user had to own an Android smartphone and to know how to operate it. A total of 265 surveys were 
collected during 10 days of the study (105 from Group A and 160 from Group B). Please refer to [11] for a more detailed description of data analysis.

\subsection{1-Day Sensing Campaign on Walkability}

The Walkability study is an initiative to explore the possibility of automatically evaluating how friendly an area is for walking. The concept of walkability includes issues such as infrastructure and physical access (e.g., sidewalks, street layout), the existence of places of interest to visit (e.g., market, schools, retail, recreation spaces, transit stops), and proximity to home.

The study was conducted with people who work or study at a university and thus recorded data in the surroundings of their campus. The study was replicated in three different countries. Table 2 shows some basic characteristics of the participants. In order to keep battery consumption low, sensor data were collected in periodic time intervals similar to the Tholilo study. Unlike the Tholilo study, in this sensing campaign we increased accelerometer's sample intervals, so we have a more detailed data to infer if the person is walking. The following sensor configuration was employed:

- GPS (every $30 \mathrm{~min})$,

- Accelerometer (sample with a frequency according to user's device configuration on intervals of $20 \mathrm{~s}$ every $5 \mathrm{~min}$ ).

Table 2. Sample characteristics.

\begin{tabular}{l|l|l|l}
\hline & Group AA & Group BB & Group CC \\
\hline No. of participants & 29 & 21 & 15 \\
\hline City, Country & Ensenada, Mexico & Istanbul, Turkey & $\begin{array}{l}\text { Toledo \& Castille-La } \\
\text { Mancha, Spain }\end{array}$ \\
\hline Size of the city & Medium $(500 \mathrm{k})$ & Large $(14 \mathrm{M})$ & $\begin{array}{l}\text { Small }(84 \mathrm{k}) \& \text { Large } \\
(2 \mathrm{M})\end{array}$ \\
\hline Density of population & $9 / \mathrm{km}^{2}$ & $2.6 \mathrm{k} / \mathrm{km}^{2}$ & $362 / \mathrm{km}^{2} \& 27 / \mathrm{km}^{2}$ \\
\hline Gender & $(15 \mathrm{male} ; 14$ female $)$ & $(17 \mathrm{male} ; 4$ female $)$ & $(4 \mathrm{male} ; 9$ female $)$ \\
\hline Average age (S.D.) & $28.48(5.79)$ & $23.24(3.62)$ & $28.42(9.08)$ \\
\hline
\end{tabular}

Each participant received a survey under two conditions: (1) automatically triggered by detecting that the participant had walked for 5 min continuously, or (2) on demand. Surveys focused on evaluating walkable areas and pedestrian experience. Data were collected during a 21-day period with an optional extension of 7 days.

Participants carried their smartphones as usual. We replicated the study by providing the same training protocol and directions to three different groups of participants. A total of 65 volunteers participated in the study. Each user owned and regularly used an Android smartphone. A total of approximately 500 surveys were completed during the study.

At the time of this writing, the collected data from the study "Walkability: a 21-day sensing camping", has not been yet analyzed in full detail. 


\section{Lessons Learned and Discussion}

Over the course of 10 to 21 days, with 77 users participating in two different studies, we have observed four main aspects related to the following issues: User-experience, technical mobile phone aspects, monitoring data, and replication of studies.

User-experience: Our main challenges developing a platform to be deployed on the user's personal mobile phone were the heterogeneity of OS version and library/services available. Across the studies, we deployed several updates to fix on-the-fly issues that did not show in our pilot test. This aspect is challenging when talking about mobile phones because users tend to rely on mobile phones for critical communication functions, such as emergency calls. Thus, we should guarantee that participants enable automatic updates-mechanism in their smartphones, so we could provide them unexpected updates along the study.

Technical mobile phone aspects: Several cases showed heterogeneous software resources and functionalities available on participants' mobiles devices due to either OS version or device model/brand. A few common examples are:

- Google Cloud Messaging (GCM): not all devices had enabled this service which offers to send messages to users through push notifications function.

- Acceleration frequency: devices have a different acceleration frequency limited by hardware sensor or set up by the user through configuration preferences.

- Linear acceleration: not all devices might provide this service due sensor requirements.

- Timestamp issue: timestamp values seems to be retrieved differently from device to device. For instance, some devices populate timestamp with nanoseconds while other use seconds (Unix time). Some sensor's model/brands include an offset to all timestamp. Others, use boot time as base for their timestamp.

We found this aspect to be important since one of the motivations of the sensing campaigns was to create open datasets accessible to the research community in an effort to provide a resource for the analysis on behavior recognition. Thus, there is a need to address the issue of data quality in heterogeneous datasets.

Monitoring data: To supervise data completeness during the campaigns, we assigned a single researcher to monitor data completeness. If a lack of data was identified, he communicated with the remote collaborator to establish communication with respective participants. In order to facilitate effort and coordination among researchers involved in multi-institutional campaigns, a controlled access to monitor data completeness is required for each collaborator. Thus, we considered expanding $\mathrm{m}^{\mathrm{k}}$-sense with a multilevel privilege section.

Replication of studies: We prepared a study protocol and closely provided support to the collaborators. Due to the effort invested when supervising campaigns remotely, we have considered to extend the current platform version with a protocol module, so we are able to tackle two main aspects: sequence of tasks and feedback among collaborators to share experiences and unexpected participant reactions that might vary from country 
to country. In addition, two aspects we had to deal with were the differences in time zones (among countries and when traveling within the country) and the different survey languages for the different samples (i.e., Spanish, Turkish, English). Thus, we collect time zone data from participant's smartphones, as well as based on geographic location. Finally, the respective client-side GUI resource was validated for 3 native speakers from each country involved in the study. The common language we used to coordinate was English.

\section{Conclusion and Future Work}

We introduced $\mathrm{m}^{\mathrm{k}}$-sense, a research initiative to facilitate the deployment and supervision of multi-institutional sensing campaigns. Principles of the platform were based on an approach-centered design, modulation by study-package, and creation of mobile phone campaigns. The architecture consists of a three-layer client-server model. The main contributions of $\mathrm{m}^{\mathrm{k}}$-sense consisted of (1) the package-study feature, (2) data completeness monitor in real-time, and (3) a graphical interface to manage sensing campaigns. Altogether, contributions focused on reducing the barrier of technical concepts for non-technical researchers, so that they could focus on analyzing data rather than investing time developing a sensing platform from scratch.

We conducted two multicultural sensing campaigns in four different countries (i.e., Turkey, Mexico, Switzerland, and Spain): (1) Thought and Life Logging, and (2) a 21day sensing campaign on walkability. A total of 12 and 65 participants collaborated in the studies. Along these campaigns we faced four relevant aspects:

- User-experience: users tend to rely on mobile phones for critical communication functions, like emergency calls, thus, a mechanism to guarantee uninterrupted support during sensing campaigns was included in the protocol, nevertheless, we would like to guarantee it programmatically into the $\mathrm{m}^{\mathrm{k}}$-sense application.

- Technical mobile phone aspects: due the heterogeneity and diversity of smartphones among participants, we will address a new mechanism when collecting data to guarantee high quality in heterogeneous datasets.

- Monitoring data: to improve coordination among multiple collaborators involved in a sensing campaign, we consider it important to include a multilevel privilege section in further versions.

- Replication of study: to ensure that a campaign's protocol is appropriately attended, we consider it is important to extend the current version with a new module to keep control of times and sequence of tasks, in which collaborators are able to create a personal schedule of activities, as well as provide/get feedback in real-time from the experience from collaborators in different locations.

Regarding technical aspects, we will consider migrating our current database core to MongoDB ${ }^{4}$ since in recent years it has shown a high reliability and better performance when working with largest amount of data.

\footnotetext{
${ }^{4}$ https://www.mongodb.com/.
} 
Additionally we aim at developing an $\mathrm{iOS}^{5}$ version of the client-side to facilitate recruitment process when designing a sensing platform. Thus, we would aim to cover a broader sample of potential participation of smartphone users and increase the sample of data collected.

Acknowledgment. We thank the participants of the two sensing campaigns described in the paper for contributing their time and effort in making them successful. This work was partially funded by (1) the Co-Funded Brain Circulation Scheme Project "Pervasive Healthcare: Towards Computational Networked Life Science" (TÜBITAK Co-Circ 2236, Grant agreement number: 112C005) supported by TÜBİTAK and EC FP7 Marie Curie Action COFUND and (2) the EC FP7 Marie Curie Action "UBIHEALTH - Exchange of Excellence in Ubiquitous Computing Technologies to Address Healthcare Challenges" (Project number 316337).

\section{References}

1. The Economist. Planet of the phones, February 2015. http://goo.gl/qoX2cc

2. TC Media. Google announces 1.4 billion Android activations, September 2015. http://goo.gl/ buxpwO

3. Silva, T.H., Vaz de Melo, P.O.S., Almeida, J.M., Salles, J., Loureiro, A.A.F.: Revealing the city that we cannot see. ACM Trans. Internet Technol. 14, 1-23 (2014)

4. Eagle, N., (Sandy) Pentland, A.: Reality mining: sensing complex social systems. Pers. Ubiquitous Comput. 10, 255-268 (2005)

5. Bengtsson, L., Lu, X., Thorson, A., Garfield, R., von Schreeb, J.: Improved response to disasters and outbreaks by tracking population movements with mobile phone network data: a post-earthquake geospatial study in Haiti. PLoS Med. 8, e1001083 (2011)

6. Brownstein, J.S., Freifeld, C.C., Madoff, L.C.: Digital disease detection-harnessing the Web for public health surveillance. N. Engl. J. Med. 360, 2153-2155, 2157 (2009)

7. Das, T., Mohan, P., Padmanabhan, V.N., Ramjee, R., Sharma, A.: PRISM: platform for remote sensing using smartphones. In: Proceeding of 8th International Conference Mobile Systems, Applications, and Services, MobiSys 2010, pp. 63-76 (2010)

8. Nandugudi, A., Maiti, A., Ki, T., Bulut, F., Demirbas, M., Kosar, T., Qiao, C., Ko, S.Y., Challen, G.: PhoneLab: a large programmable smartphone testbed. In: Proceedings of First International Workshop on Sensing and Big Data Mining, pp. 1-6 (2013)

9. Froehlich, J., Chen, M.Y., Consolvo, S., Harrison, B., Landay, J.A.: MyExperience: a system for in situ tracing and capturing of user feedback on mobile phones. In: Proceedings of the 5th International Conference on Mobile Systems, Applications and Services, pp. 57-70. ACM, New York (2007)

10. Aharony, N., Gardner, A., Sumter, C., Pentland, A.: Funf: Open Sensing Framework (2011). http://funf.media.mit.edu

11. Hernández, N., et al.: Thought and life logging: a pilot study. In: García-Chamizo, J.M., Fortino, G., Ochoa, S.F. (eds.) UCAmI 2015. LNCS, vol. 9454, pp. 26-36. Springer, Heidelberg (2015). doi:10.1007/978-3-319-26401-1_3

\footnotetext{
${ }^{5}$ http://www.apple.com/ios/.
} 